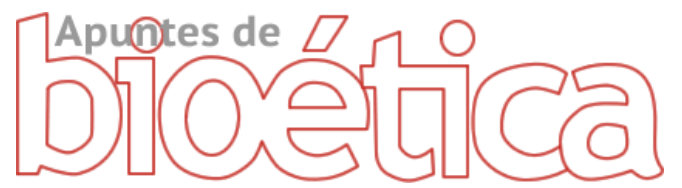

https://doi.org/10.35383/apuntes.v4i2.657

e-ISSN:2663-4910

\title{
La relación médico paciente vista desde la experiencia de los familiares de enfermos con cáncer en etapa terminal
}

\author{
Doctor-patient relationship seen from the experience of relatives of \\ terminal cancer patients
}

\author{
Norma Alicia Ordóñez-Vázquez ${ }^{*}, 1$, a \\ camel1806@yahoo.com.mx \\ https://orcid.org/0000-0002-1722-6546
}

Zuraya Monroy-Nasr ${ }^{*}, 1, \mathrm{~b}$

zuraya03@gmail.com

https://orcid.org/0000-0002-3837-5735

\author{
*Autor corresponsal \\ ${ }^{1}$ Universidad Nacional Autónoma de México, Ciudad \\ de México, México \\ a Doctora en Ciencias de la Salud \\ ${ }^{\mathrm{b}}$ Doctora en Filosofía
}

Fechas importantes

Recibido: 28/09/2021

Aceptado: $21 / 12 / 2021$

Publicado online: $23 / 12 / 2021$

\begin{abstract}
Resumen
La relación médico-paciente es un elemento importante del ejercicio profesional del médico y por lo tanto de la ética médica. Crear una buena relación interpersonal entre médico y paciente facilita la comunicación mediante una interacción empática, esto ayuda a tomar decisiones al final de la vida y respetar la autonomía. La finalidad de este trabajo es conocer, desde de la visión de los familiares de enfermos con cáncer en etapa terminal, la relación que mantuvo el galeno con el paciente a partir de los tipos de relación médico-paciente. Se llevó a cabo una metodología cualitativa, utilizando el paradigma hermenéutico fenomenológico. Se entrevistaron once familiares y cuidadores primarios y se utilizó el análisis temático y el método de van Manen. Los resultados muestran que la relación que establecieron los médicos fue diferente dependiendo del tipo de hospital. Esto permite conocer cómo actuaron los médicos a partir de la beneficencia, el respeto a la autonomía, y la no maleficencia en su relación con los pacientes. Es importante el estudio de esta relación desde diversas disciplinas para encontrar áreas de mejora en la atención del paciente terminal.
\end{abstract}

Palabras clave: Autonomía; Beneficencia; Ética Médica; Fenomenología Hermenéutica; Paternalismo; Relación Médico-Paciente. 


\begin{abstract}
The doctor-patient relationship is an important element of medical practice and therefore of medical ethics. Creating a good interpersonal relationship between doctor and patient facilitates communication through empathic interaction, which helps in proper decision making at the end of life and respect for autonomy. The objective of the article is to know, from the experience of the relatives of terminalstage cancer patients, the relationship that the doctor maintained with the patient based on the doctor-patient relationship models. A qualitative study was carried out that starts from the phenomenological hermeneutical paradigm. Eleven family members and primary caregivers were interviewed and the thematic analysis and van Manen method were used. The results show that the relationship established by the doctors was different depending on the type of hospital. This allows us to know how doctors acted from beneficence, respect for autonomy, and non-maleficence in their relationship with patients. The study of this relationship from various disciplines is important to find areas for improvement in terminal patient care.
\end{abstract}

Keywords: Autonomy; Beneficence; Medical Ethics; Hermeneutical Phenomenology; Paternalism; Doctor-Patient Relationship

\title{
Introducción
}

La relación médico-paciente es la base de la práctica médica y de la ética que guía el comportamiento de los médicos (Williams, 2005). Crear una buena relación interpersonal entre médico y paciente facilita una comunicación adecuada, en la que es importante la información que se emite, con detalles y que sea fácilmente comprensible. Esta relación se refiere al trato que el médico establece con el paciente en la que van a presentarse implicaciones éticas, filosóficas, psicológicas y sociológicas que existen en la práctica profesional en el ámbito de la salud (González, 2004). No se debe olvidar en esta relación médico-paciente la empatía, trato humanitario y sensible.

Existen distintos tipos de relación médico-paciente en los que el médico interactúa con el paciente y transmite la información (Emannuel y Emannuel, s.f.). Estos tipos de relaciones muestran la forma en que el médico actúa frente a las características de cada paciente, y conforme con sus valores o decisiones. Es preciso señalar que lo principal en cada tipo de relación es el respeto de la autonomía y evitar la maleficencia.

Paternalista. Este tipo de relación prevaleció varios años en la que el médico fungía como la principal figura en la que no se tomaba en cuenta la opinión ni decisión el individuo. Actualmente existe una mayor participación del paciente en la toma de decisiones respecto a tratamientos e intervenciones (Cañete, Guilhem y Brito, 2017).

Esta relación es concebida como paternal, en la que el médico establece a partir de sus conocimientos, la toma de decisiones en la que considera que el paciente recibe la mejor 
atención y los tratamientos que van a proteger su salud y tranquilidad. Es así que el médico dispone lo que considera es la mejor opción para el paciente y prescinde de su opinión o decisión (Emanuel y Emanuel, s.f.).

Informativo. Este tipo de relación en ocasiones se le llama técnico o del consumidor. El médico que utiliza esta relación establece una comunicación participativa con el paciente, en la que facilita toda la información respecto a su diagnóstico y tratamiento, esto permite que pueda elegir lo que más se ajuste a sus decisiones. Los pacientes que están bien informados presentan menos incertidumbre del conocimiento de su estado de salud lo que permite que disminuya la ansiedad y el estrés.

Interpretativo. La intención de ese tipo de relación se basa en que el médico ayuda al paciente a seleccionar qué intervención es la que más se relaciona con sus valores. También se proporciona información detallada sobre el estado de salud, los medicamentos y procedimientos médicos a realizar, como en el tipo de relación informativa. Otra de las ventajas de esta relación es que el médico proporciona ayuda al paciente para disipar dudas y elegir lo que más se acomode a las necesidades y valores.

Deliberativo. En esta relación médico-paciente se privilegia la reflexión y el compromiso para llegar a una toma de decisiones de acuerdo con lo que mejor se ajusta a la situación del paciente. De la misma forma que en los anteriores tipos de relación, se proporciona información detallada, se ayuda a la toma de decisiones y se actúa con empatía y consideración hacia el paciente. El médico va a mostrarse como un amigo, señala las opciones adecuadas que pueden ayudar de mejor manera el pronóstico del paciente, respeta sus decisiones, transmite comprensión y ayuda en la deliberación de la toma de decisiones (Mejía y Romero, 2017).

Los diferentes tipos de relación médico-paciente se van a relacionar con una buena empatía, trato, y una toma de decisiones informada. Entonces, se puede concebir como una adecuada relación el transmitir información relevante, el detallar tratamientos apropiados, el respeto a la autonomía, y el facilitar un trato amable. En la relación con los médicos debe prevalecer un ambiente de confianza en donde el paciente sienta seguridad de transmitir sus dudas, temores y síntomas que le pueden causas ansiedad. Igualmente, es necesario que se interese en sus pacientes, pues cada caso es diferente, debe mostrar atención en el caso, 
recomendando los tratamientos que se ajusten a los diagnósticos y pronósticos, siempre prevaleciendo la beneficencia hacia el paciente.

Las características antes mencionadas de la relación médico-paciente son las que deben establecerse para lograr una adecuada atención que impactará de forma positiva en el tratamiento y su apego a éste. Cada uno de estos tipos de relación médico-paciente muestra una forma diferente de tratar al paciente, esto va a depender de diversos factores como tanto de la personalidad del médico como del paciente y familiares. Por lo tanto, no se puede hablar de una relación ideal, pues se pueden conjuntar varios aspectos de cada tipo de relación para así garantizar que se cubran las necesidades de los pacientes.

Es así que el estado emocional y la manera en que enfrenten la condición el enfermo y la familia se determinarán en gran medida con base en la forma de interacción con el médico. Una condición necesario para una buena toma de decisiones es la actuación del médico para brindar orientación y apoyo, tanto al enfermo como a la familia, respecto a la evolución de la enfermedad, siempre proporcionando respeto a la autonomía, condescendencia, confidencialidad, empatía, y considerar en todo momento la participación el enfermo, estas características fomentarán un mejor apego al tratamiento y a la reflexión de los deseos al final de la vida (Sánchez y Contreras, 2014, Arzate, Ochoa y Alvarado, 2013, Montes, Morillo, Navarro, Herminia, Querd y Pere, 2019).

Los trabajos llevados a cabo por Jiménez, Rivera, Barrera, Manuell, De la Garza, Torres y Tena, 2002, Haumi, Fuentes, Aguirre, Ramírez, 2012, Chá, 2020 y Fritz, 2020, coinciden en que la relación médico-paciente actualmente ha cambiado. Debido a la saturación de trabajo difícilmente se establecen lazos de confianza entre el médico y sus pacientes. Las consultas se realizan de manera rápida, se nombra a los pacientes por sus números de afiliación y no por sus nombres $y$, dada la premura, en ocasiones no se comunica de manera adecuada la información al paciente o la familia.

Como se puede observar el estudio de la relación médico paciente es relevante ya que permite visualizar cómo está actuando y tratando el médico a los pacientes, si respeta su autonomía, es beneficiente y proporciona información suficiente sobre el diagnóstico y pronóstico. El propósito del trabajo es entender y analizar la experiencia de los cuidadores y familiares del enfermo en fase terminal de cáncer, en cuanto a la manera en la que el médico estableció la relación con los pacientes al final de la vida. Esto se realizó a partir de entrevistas en profundidad, las cuales proporcionaron información sobre la forma en que los 
médicos establecieron relación con los pacientes en diferentes hospitales y la dinámica que se llevó a cabo.

\section{Material y métodos}

\section{Tipo de estudio}

Se llevó a cabo una investigación cualitativa centrada en el paradigma hermenéutico fenomenológico. Este modelo posibilitó explorar y entender las vivencias cotidianas de los individuos (Van Manen, 1990), es decir, la visión de los familiares del paciente con enfermedad terminal respecto a la relación del enfermo con el médico. La fenomenología ha sido utilizada en varios estudios del campo de la salud, tratando de encontrar una interpretación a las experiencias de las personas. De acuerdo con esto, Dickinson (2004) indica que el investigador va a encontrar los significados ocultos o sin importancia por las personas, y que son importantes para darse cuenta de las situaciones que no se perciben y son relevantes en su vida y acciones habituales.

La fenomenología ha sido considerada como un método de investigación que se relaciona con el estudio de la filosofía (De los Ríos y Ávila, 2004). A partir de esta disciplina se entendió y analizó la experiencia o vivencia de la enfermedad desde los argumentos de familiares de enfermos. Desde este marco teórico se han realizado algunos estudios en el ámbito de la salud, como en la enfermería, medicina, trabajo social. Los estudio se han centrado en temas como la experiencia del dolor y sufrimiento, la enfermedad, los cuidados paliativos, atención de la salud, la relación médico paciente familiares, así como con otros temas relevantes (Rodríguez, 2008, Montoya Schmidt, García-Caro, Guardia, Cabezas y Cruz, 2010).

\section{Participantes}

Se entrevistaron siete cuidadores primarios y cuatro familiares de enfermos, algunos de ellos fueron tratados en hospitales del gobierno (cinco pacientes) y otros en hospitales particulares en los que pagaron sus tratamientos (dos pacientes).

En este trabajo se utilizó el muestreo por conveniencia porque se trabajó con una muestra de informantes voluntarios que fueron captados por varios porteros. La elección de los participantes obedeció a la accesibilidad que se tuvo para su contacto más que por el propósito teórico del conocimiento inicial del fenómeno de estudio (Martínez, 2012). 


\section{Instrumento}

La guía que se elaboró para la entrevista estuvo compuesta por cinco ejes temáticos. Se indagó la vivencia de los familiares de los enfermos en los diversos procesos de enfermedad y atención por parte de los médicos. Se elaboró esta guía con base en los propósitos del estudio y la consulta de diversas fuentes. Este trabajo se desprende de una investigación más amplia, por lo que en este artículo se toma la información del eje temático: 4. Relación médico paciente, en el cual se encuentra el tema relación médico paciente y el subtema adecuada comunicación. En la siguiente tabla se muestran los temas que ayudaron a dirigir la entrevista:

Tabla 1

Ejes temáticos de la guía de entrevista semiestructurada

1. El inicio de la enfermedad, darse cuenta de ésta.

2. Principales necesidades durante la enfermedad.

3. El tránsito por los servicios de salud y la atención del médico.

4. Relación médico paciente

5. Las decisiones para tener una muerte digna, preparación para ésta.

Nota: Elaboración propia a partir de los temas y subtemas que se obtuvieron de las entrevistas que se realizaron.

\section{Procedimiento}

Se inició entrevistando a 11 individuos que accedieron a participar. Es importante señalar que ocho integraron díadas, colaborando cuidador primario y familiar, y únicamente tres cuidadores primarios que señalaron que sus familiares no aceptaron cooperar. El principal objetivo de las díadas fue complementar la información proporcionada por el cuidador.

Algunas de las entrevistas, específicamente seis, se realizaron en un sitio sin ruido que fue proporcionado por la institución pública especializada en el tratamiento del cáncer, y cinco en algunos lugares libres de ruido e interrupciones. Los informantes proporcionaron su consentimiento para ser audiograbadas las entrevistas, y se les preguntó si deseaban utilizar un seudónimo. Sin embargo, para garantizar la confidencialidad de los participantes se eligió identificarlos con números. 
El proceso que se llevó a cabo después de la transcripción de las entrevistas fue utilizar el análisis temático y el método de van Manen para comenzar a examinar la información. La característica principal de este método es realizar una exploración prudente de los que se está leyendo, de esta forma se puede encontrar la esencia de la experiencia. Para lograrlo, se tiene que realizar una detenida lectura de las transcripciones, verificar que lo que se dice concuerde con lo que se transcribió, y así tener la información precisa de la entrevista. (Van Manen, 1990). En la tabla 2 se muestran los temas y subtemas que emergieron del análisis de las entrevistas, a partir de este método de análisis:

Tabla 2

Temas y subtemas

\begin{tabular}{|l|l|}
\hline Tema & Subtemas \\
\hline $\begin{array}{l}\text { 1. Experiencia del proceso de } \\
\text { enfermedad }\end{array}$ & $\begin{array}{l}\text { Inicio de la enfermedad } \\
\text { Tratamiento } \\
\text { Evolución de la enfermedad } \\
\text { Papel de la familia (redes de apoyo) } \\
\text { Reacciones de los familiares }\end{array}$ \\
\hline $\begin{array}{l}\text { 2. Cuidados y atención a las } \\
\text { necesidades del enfermo }\end{array}$ & $\begin{array}{l}\text { Cuidados paliativos: necesidades médicas, psicológicas, } \\
\text { sociales y espirituales de los enfermos } \\
\text { El médico como apoyo al enfermo y a la familia }\end{array}$ \\
\hline 3. Relación médico-paciente & $\begin{array}{l}\text { Beneficencia del médico en el tratamiento } \\
\text { Adecuada comunicación }\end{array}$ \\
\hline 4. Decisiones al final de la vida & $\begin{array}{l}\text { Respeto a la autonomía } \\
\text { El enfermo o la familia como tomadora de decisiones }\end{array}$ \\
\hline
\end{tabular}

Nota: Elaboración propia a partir de los temas y subtemas que se obtuvieron de las entrevistas que se realizaron.

Para llevar a cabo el trabajo se sometió y fue aceptado por la Comisión de Investigación y Ética del Instituto público que proporciona tratamiento a pacientes con cáncer.

\section{Resultados}

Como se comentó anteriormente, se entrevistaron 11 personas, las cuales fueron familiares y cuidadores de los enfermos con enfermedad terminal. En las siguientes tablas se especifican las particularidades de los participantes. 
Tabla 3

Características de familiares de enfermos con cáncer terminal

\begin{tabular}{|c|c|c|c|}
\hline Familiares & Edad & Escolaridad & Parentesco con el enfermo \\
\hline $\begin{array}{l}11 \text { participantes } \\
8 \text { formaron díadas } \\
\text { cuidador/familiar }=(4 \\
\text { cuidadores } / 4 \\
\text { familiares }) \\
3 \text { cuidadores primarios }\end{array}$ & 16-76 años & $\begin{array}{l}3 \text { educación básica } \\
2 \text { preparatoria } \\
6 \text { licenciatura } \\
1 \text { posgrado }\end{array}$ & $\begin{array}{l}4 \text { hijas } \\
2 \text { esposos/1 esposa } \\
1 \text { madre } \\
3 \text { hermanas }\end{array}$ \\
\hline
\end{tabular}

Nota: Elaboración propia a partir de las entrevistas que se realizaron a los familiares de los enfermos.

Tabla 4

Características de los enfermos con cáncer terminal

\begin{tabular}{|l|l|l|}
\hline \multicolumn{1}{|c|}{ Enfermos } & \multicolumn{1}{|c|}{ Tipo de cáncer } & \multicolumn{1}{c|}{ Tipo de hospital } \\
\hline $\begin{array}{l}7 \text { enfermos en total } \\
4 \text { enfermos }=4 \text { cuidadores/4 } \\
\text { familiares } \\
3 \text { enfermos=3 cuidadores }\end{array}$ & $\begin{array}{l}\text { 1 piel basocelular (se } \\
\text { encontraba en remisión) } \\
2 \text { cáncer de mama } \\
1 \text { cáncer de esófago } \\
1 \text { tumor cerebral } \\
1 \text { leucemia } \\
1 \text { ovario }\end{array}$ & $\begin{array}{l}\text { atención de cáncer } \\
3 \text { Público } \\
1 \text { Privado/Instituto especializado } \\
\text { en atención de cáncer } \\
1 \text { privado }\end{array}$ \\
\hline
\end{tabular}

Nota: Elaboración propia a partir de las entrevistas que se realizaron a los familiares de los enfermos.

Relacionado con este eje se desprende el subtema adecuada comunicación. Aunque en este artículo solamente se describe la información de la relación médico paciente es necesario comentar la comunicación brevemente, al formar parte importante de esta relación y de los resultados obtenidos.

De los resultados relacionados con el subtema "adecuada comunicación" que forman parte del tema relación médico-paciente se encontró lo siguiente. La experiencia que tienen los familiares de la comunicación médico-paciente fue diversa. El panorama que muestran de este proceso de enfermedad y la comunicación indica que los médicos presentaban renuencia a hablar con el paciente y la familia de un mal diagnóstico. La información que algunos recibieron fue escasa o insuficiente, por ejemplo, cuando no se quiso comunicar el avance del padecimiento, exagerada al dar información en la que se realzaron los síntomas, reducida o somera para no tratar el tema de la muerte, y confortante para apoyar al paciente y a los familiares de un diagnóstico desalentador. Se tiene que recordar que en la relación médico paciente es relevante contar con una atención personalizada y un vínculo 
interpersonal apropiado en donde el médico permita una comunicación adecuada, es decir, se intercambie información, y se logre transmitirla con detalles y sea fácilmente comprensible.

Tabla 5

Experiencia de los familiares de los enfermos con cáncer terminal respecto a la comunicación médicopaciente

\begin{tabular}{|c|c|c|}
\hline Escasa o insuficiente & $\begin{array}{l}\text { Información } \\
\text { sobredimensionada }\end{array}$ & Información alentadora \\
\hline $\begin{array}{l}\text {... y fue el primer doctor que } \\
\text { me dijo "iA tu hermana le } \\
\text { queda un mes de vida!" ... Sí } \\
\text { me dijo, fue el único, que me } \\
\text { dijo a mí y porque yo fui y le } \\
\text { pregunté, y le dije doctor, } \\
\text { quiero hablar con usted, era el } \\
\text { que estaba escribiendo y no le } \\
\text { levantaba la vista para ver a } \\
\text { mi hermana...Quisiera } \\
\text { conservar más a mi hermana, } \\
\text { ¿puede decirme?, "si le va } \\
\text { bien dura tres meses, si le va } \\
\text { mal le dura un mes", le digo } \\
\text { ¿Y si no le pone quimio?, dice } \\
\text { "no pasa de este mes" ... } \\
\text { Participante } 1 \text { (familiar). }\end{array}$ & $\begin{array}{l}\text {...estuvo en oncología como una } \\
\text { semana, nosotros la ingresamos } \\
\text { con la idea simplemente de que la } \\
\text { estabilizaran un poco y luego } \\
\text { llevarla a casa, pero ya cuando } \\
\text { estábamos ahí se empezó a } \\
\text { dificultar el asunto porque en } \\
\text { oncología nos decían "en su casa } \\
\text { no la van a poder tener bien } \\
\text { como la tenemos aquí" porque } \\
\text { cuando llegamos le pusieron } \\
\text { alimentación artificial, tenía el } \\
\text { oxígeno y cuando lo necesitaba se } \\
\text { lo ponían, y nos dijeron los } \\
\text { médicos que en la casa se } \\
\text { pondría muy mal. Participante } 2 \\
\text { (cuidadora). }\end{array}$ & $\begin{array}{l}\text {...y el doctor habla con él, se } \\
\text { encierra y le dice: "por favor } \\
\text { vuelve a la quimio puedes } \\
\text { vivir más tiempo y con más } \\
\text { calidad con quimio", y } \\
\text { después de que había } \\
\text { tomado la decisión, habían } \\
\text { pasado dos meses sin quimio } \\
\text { pues lo convenció el doctor, } \\
\text { por las ganas de vivir. } \\
\text { Participante } 3 \text { (cuidadora). }\end{array}$ \\
\hline
\end{tabular}

Nota: Elaboración propia a partir de las entrevistas que se realizaron a los familiares de los enfermos.

Se presenta en estas experiencias de los familiares entrevistados, que los médicos procedieron de acuerdo a la beneficencia del paciente, pero en el caso de la participante 1 y 2 se sigue un modelo paternalista, al no comunicar el pronóstico de la enfermedad, no respetaron la autonomía del enfermo al no proporcionarle información, someterlo a tratamientos desproporcionados y dar información sobredimensionada a la familia. El tipo de relación médico-paciente informativo se observó muy poco, debido a que los pacientes no recibieran información apropiadamente para así tomar decisiones sobre los medicamentos o tratamientos.

Se muestra en la tabla 6 algunas de las experiencias de la relación paternalista e informativa que se identificaron en el análisis de las experiencias de algunos familiares: 
Tabla 6

Modelos médicos que ejemplifican la comunicación en la relación médico-paciente

\section{Modelo paternalista}

Nunca fue una opción decirnos "Quieren que reciba alimentación ¿o no?" fue como parte de su protocolo, ahora lo veo que eso no debió pasar así seguramente mi hermana de entrada hubiera dicho "No quiero sonda" pero no se lo preguntaron. El trato era más humanitario, pero aún en ese contexto me parece que la voz del paciente no era atendida. Yo creo que ahí pasa algo con los pacientes moribundos, creo que los médicos dan por hecho que ya no son capaces de nada cuando no es así, te estoy hablando de un paciente consciente que sí está debilitado, que sí está cansado y que sí todo eso sí, pero tiene plena conciencia y los médicos no sé si por su propio temor o por esta tradición rara de que los enfermos ya no son personas completas no los toman en cuenta, ino sé! Todavía no me explico porque hacen esta omisión de los "murientes" a nosotros no se nos preguntó si le ponían o no sonda. Nosotros llegamos pidiendo estabilícenla, no queremos que tenga dolor, no queremos que muera asfixiada, pero de ahí a que se nos explicara cuáles eran las consecuencias o no de hacer tal o tal cosa, no. Participante 2 (cuidadora).

Nota: Elaboración propia a partir de las entrevistas que se realizaron a los familiares de los enfermos.

Lo que se interpreta de la vivencia de la cuidadora (participante dos), es que los médicos no consideraron la autonomía de la enferma, que aun podía comunicar sus preferencias y sus familiares estaban ahí para apoyarla, porque realizaron las intervenciones (aplicación de la sonda) sin su consentimiento. En este caso la paciente estaba consciente para emitir sus decisiones que no fueron respetadas. No obstante, los médicos actuaron a partir del modelo paternalista, en el que pretendían ayudarla a seguir alimentándose, ya que tenía problemas para comer. No preguntaron cuál era su decisión, por lo que no tomaron en cuenta sus preferencias y voluntades de cómo deseaba morir. En la Ley General de Salud (2020) en el artículo 166 Bis 18 se especifica que "para respaldar la calidad de vida del enfermo en condición terminal, el médico tiene que dejar de utilizar medidas extraordinarias que son consideradas como encarnizamiento terapéutico". En este caso, se puede visualizar que se estaban adoptando medidas desproporcionadas ya que la paciente era terminal y su decisión 
era ya no recibir tratamiento.

En los últimos veinte años, el interés en estudiar la relación médico paciente ha estado enfocada en lo que es el paternalismo y la autonomía. La relación médico paciente paternalista se centraba en que el médico era el especialista que contaba con los conocimientos y que el paciente tenía que acatar sus determinaciones, por ser lo mejor para éste. Este tipo de relación ha sido discutida porque puede llevar a un autoritarismo (Moreno, 2019), como se puede ver en esta experiencia.

En el caso de la participante tres y cuatro, la experiencia que comparten presenta que el tipo de relación del médico con los pacientes fue informativo, aunque pueden encontrarse características del tipo de relación interpretativo e incluso del deliberativo. El médico se comportó de forma amable, empática, transmitió información adecuada sobre tratamientos y proporcionaba alternativas para que tuvieran tranquilidad tanto el paciente como la familia. Es así que el enfermo, al contar con información de su pronóstico elaboró sus voluntades y murió como lo decidió. En este caso el paciente fue atendido en un hospital privado. Es relevante indicar que las diferencias sector público y privado son evidentes, de acuerdo con el estudio de Souza, Gomes, Dos Santos, Machado y Machado (2020) se encontró que los pacientes de los servicios privados de salud se sintieron mejor informados acerca de su patología, lo que puede estar relacionado con una mayor exigencia y expectativa.

Estas experiencias permiten observar, sin realizar generalizaciones, que en el servicio de salud pública existió un predominio del tipo de relación paternalista, en la que las determinaciones son tomadas por el médico sin considerar la voz del paciente. Por el contrario, en la experiencia del hospital privado, se observa un modelo informativo. Esta información puede dar cuenta de que no se está cumpliendo con el principio bioético de justicia, en donde no deberían existir diferencias en el trato y cuidado del enfermo con enfermedad terminal, a pesar de que es evidente que hay muchos determinantes sociales que afectan la atención a la salud e impiden que haya un sistema de salud público de calidad. El hecho de no pagar por un servicio de salud no debería ser una condición para recibir un trato más humano y empático al final de la vida. No obstante, es una condición que impera actualmente en nuestro sistema de salud. 


\section{Discusión}

La relación médico paciente es una relación interpersonal en la que hay interacción entre los enfermos y los médicos, de esta forma el galeno tiene que proporcionar información en la que tiene que demostrar interés y empatía en el padecimiento del enfermo.

Se ha señalado de manera constante que la relación médico-paciente es una relación asimétrica, una relación en la que entra en juego el poder. Planteado de esta manera, cobra sentido la denominación de "facultativo" dada a quien se le otorga el permiso de entrar en la intimidad de otra persona, de presenciar su debilidad, y de quien se espera ayuda y servicio, puesto que ha sido considerado con suficientes conocimientos, más prudente, más entendido en materia de sufrimiento humano, pues ha recibido la preparación necesaria. Además, con cierta frecuencia, los médicos son percibidos con estas expectativas por parte de sus pacientes, quienes les atribuyen, asimismo, consciente o inconscientemente, cualidades y valores como la solidaridad, la compasión y el respeto, por mencionar algunos ejemplos (Hernández y Jouve, 2020).

De acuerdo con lo anterior es fundamental la comunicación del médico con el enfermo, pues a partir de ésta el enfermo se siente acompañado, comprendido, tratado dignamente al ser considerado para decidir sobre tratamientos o procedimientos médicos, que garantiza el respeto a la autonomía y beneficencia (Torío y García, 1997; Makoul, Arntson y Schofield, 1995).

La vivencia de los familiares que se analizó, muestra hubo problemas para transmitir información sobre el avance de la enfermedad, procedimientos médicos, al otorgar información exagerada de los síntomas de los pacientes, lo que generó ansiedad los enfermos y familiares. Además, la información sobre el progreso de la enfermedad la ofreció el médico al cuidador o los familiares, sin considerar al paciente que contaba con capacidad cognitiva para entender la información y emitir su decisión.

Se puede apreciar a partir de la experiencia de los familiares, que se presentan dos tipos de relación médico-paciente. Por una parte, el tipo de relación paternalista, en la que prevalece tomar decisiones en pro del bienestar del paciente sin otorgar información del pronóstico, medicamentos y tratamientos; ni contemplar la autonomía del paciente para tomar sus decisiones. El otro tipo de relación es el informativo, que se presentó en el hospital privado, y que se diferencia del paternalista, porque los médicos ofrecen información sobre el 
pronóstico, tratamientos y las voluntades anticipadas. Obtener este tipo de información permite que los enfermos tomen decisiones respecto a lo que desean al final de su vida.

Por lo tanto, estas experiencias permiten analizar los tipos de relaciones médico-pacientes que se presentan en el ámbito tanto público como privado. En hospitales públicos no se presentó una buena relación médico paciente, porque los médicos no tuvieron la disposición ni el tiempo para comunicar adecuadamente ni para conocer mejor al paciente, esto puede deberse a la falta de capacitación para lidiar con el paciente terminal, por la falta de tiempo debido a la saturación de pacientes que atender, o por falta de voluntad. En cambio, los enfermos que eligieron y tuvieron los recursos para ser tratados en hospitales privados, tuvieron atención personalizada, los médicos y las enfermeras fueron amistosos y empáticos tanto con el enfermo como con la familia, y se encontraban arropados en un ambiente de confianza y seguridad.

En el país la falta de formación en las escuelas de medicina sobre cómo tratar a los pacientes al final de la vida, coloca a los médicos en situaciones difíciles de enfrentar. Es muy importante la sensibilización, empatía, comunicación y trato humano en la relación de los enfermos con el médico. La beneficencia y respeto a la autonomía del paciente, es una condición necesaria para lograr un eficiente trato tanto al paciente como a la familia. Es indispensable señalar que además de un entrenamiento especial para atender a enfermos terminales, la crisis sanitaria del país no ha permitido que se logren avances significativos en esta área, y que en ocasiones los médicos hagan lo que pueden con los pocos recursos con los que cuentan.

Como indica Benito, et al., (2020) el paradigma biomédico que domina en el modelo sanitario, apenas integra la dimensión subjetiva de la persona, y las emociones y el sufrimiento no suelen ser considerados en la formación académica, a pesar de que impregnan todo el quehacer y la relación médico-paciente.

Por esta razón se hace necesario el aporte de la psicología, filosofía, la bioética y ética médica para investigar y estudiar la relación médico-paciente en sus diferentes dimensiones: ética, médica, psicológica, social y moral; fortalecer las bases éticas y legales de los médicos, lograr que sean capaces de analizar y hacer propuestas que lleven a la solución de los conflictos y dilemas que se generan con el desarrollo, la innovación del conocimiento y la aplicación de la tecnología en los seres humanos, y en todos los seres vivos (Mejía y Romero, 2017). 
Es importante aclarar que se necesitan realizar más estudios para conocer cómo es esta relación del médico con los enfermos en la atención de la salud pública y privada, ya que esto nos permitiría identificar áreas de mejora en la salud y ver la salud desde una perspectiva humana y no como un intercambio monetario.

\section{Conclusiones}

Como bien se ha señalado, la relación del médico con el paciente es la interrelación social que facilita transmitir y recibir información que ayuda a la toma de decisiones. Si existe una buena relación, una apertura por parte del médico a conocer un poco más la historia del paciente, puede darse cuenta de las necesidades y preferencias de éste, de esta forma podrá aconsejar y sugerir medidas que pueden ayudar a elegir opciones médicas que mejor se ajusten al final de la vida.

Es evidente que el sistema de salud está rebasado, en la salud pública no hay tiempo para conocer a los pacientes, hay una saturación de pacientes, rotación de médicos, aunado al burnout que aparece por el exceso de trabajo. De esta forma, como lo señala Benito et al., (2020) los médicos tienen una gran cantidad de labores que van desde lo administrativo hasta lo clínico, en donde tienen que atender a varios pacientes en poco tiempo, aunado a escuchar problemas emocionales de los enfermos, la falta de recursos y la toma de decisiones en casos de urgencias, todo esto puede afectar su rendimiento y en ocasiones reducir la empatía.

Como se puede ver, el médico tiene una gran responsabilidad, que a veces no puede manejar de la mejor forma. Es comprensible que la falta de tiempo y la presión por atender a cierta cantidad de pacientes en determinado tiempo en el sistema de salud público, interfiera para atender a los pacientes de forma más empática; no obstante, es preciso destacar que en el servicio de atención privada, el médico se comporta de manera más humana, con más interés en el paciente, así como de tratar de ayudarlo a encontrar mejores alternativas de tratamiento, como se vio en la experiencia presentada. Derivado de esto, y con base en lo que se presenta en este trabajo, es necesario cuestionarse si es necesario que la atención de salud sea de calidad solamente si se cuentan con los recursos para pagarla. Algo que debe reflexionar el médico, es el tratar con el dolor y sufrimiento del paciente, informarle que es lo mejor para él, proporcionarle alternativas de tratamiento, intentar un acercamiento, sin que esto implique involucrarse y se afecte su salud mental. 
También se debe reconocer que los médicos no cuentan con una formación que le ayude a manejar sus emociones ante el dolor y sufrimiento del enfermo terminal, ya que las escuelas de medicina no enseñan a fomentar las habilidades y recursos para estas situaciones, por lo que en ocasiones prefieren limitar la relación con los pacientes terminales.

En un estudio cualitativo realizado a médicos de la Ciudad de México y de Puebla por la Asociación Civil Por el Derecho a Morir con Dignidad (2015), reporta que los médicos señalan que las diferencias entre el trato a los pacientes en situación terminal en instituciones públicas y privadas son considerables, esto como consecuencia de que en las instituciones públicas hay mucha demanda y pocos recursos, por lo que se opta por poner atención en los pacientes que pueden recuperarse, y no atienden debidamente a los que están en una etapa terminal de la enfermedad.

Si bien este trabajo es cualitativo y no se puede llegar a generalizaciones, es indudable que se necesitan realizar más estudios en los que se analice la relación que se da entre el médico y el paciente, los modelos de relación médico-paciente que más se están utilizando en los servicios de salud públicos y privados, y así poder comprender qué es lo que sucede y qué se necesita para mejorar esta relación. Todo esto para una mejora en la atención del enfermo con enfermedad terminal y de la resolución que tome al final de la vida. 


\section{Referencias}

Arzate, M. C., Ochoa, C. J., Alvarado, A. S. (2013). La relación médico-paciente-familia en Oncología. Gaceta Mexicana de Oncología, 12 (1). 41-48. https://bit.ly/3sjiUXw

Benito, E., Rivera, R. P., Yaeguer, J., Specos, M. (2020). Presencia, autoconciencia y autocuidado de los profesionales que trabajan con el sufrimiento. Apuntes de Bioética, 3 (1), 72-78. https://doi.org/10.35383/apuntes.v3i1.399

Cañete R, Guilhem D, Brito K. (2012). Consentimiento informado: algunas consideraciones actuales. Acta Bioeth, 18 (1), 121-7. http://dx.doi.org/10.4067/S1726-569X2012000100011

Chá, G. M. (2020). Historia clínica electrónica: factores de resistencia para su uso por parte de los médicos. Rev. Méd. Urug, 36 (2), 122-143. http://dx.doi.org/10.29193/rmu.36.2.6.

De los Ríos, C. J. y Ávila, R. T. (2004). Algunas consideraciones en el análisis del concepto: satisfacción del paciente. Investigación y Educación en Enfermería. Medellín, XXI: 2.

Dickinson, R. A. (2004). The family/practitioner relationship in the context of chronic childhood illness. Thesis. Degree Doctor of Philosophy. Auckland University of Technology.

Emanuel, J. E., Emanuel, L.L. (s.f.). Cuatro modelos de la relación médico- paciente. https://bit.ly/3FhzUAR

Fritz, A. (2020). La transformación digital en la atención sanitaria como un reto para la autonomía y la confianza en la interacción médico-paciente. Revista Internacional de éticas Aplicadas, 32. https://bit.ly/3perCEv

González, M. R. (2004). La Psicología en el campo de la salud y la enfermedad. La Habana: Editorial Científico Técnico.

Hernández, S. LG. y Jouve de la Barreda, N. (2020). ¿La eutanasia como opción ante el sufrimiento? Una mirada desde la Psiquiatría. Apuntes de Bioética, 3 (1), 33-46. https://doi.org/10.35383/apuntes.v3i1.386

Huami, S.L., Fuentes, G.R., Aguirre, H. R., Ramírez de la Roche, F. O. (2012). Expectativas y experiencias de los usuarios del Sistema de Salud en México: Un estudio de satisfacción con la atención médica. México: Facultad de Medicina, UNAM.

Jiménez, C. M., Rivera, C. A., Barrera, R. N., Manuell, L, G., de la Garza, S. J., Torres, L. A., Tena, T. C. (2002). Recomendaciones para mejorar la práctica de la medicina en la atención del paciente con cáncer. Revista Conamed, 6 (11), 5-12.

Ley General de Salud. (2018). México, Cámara de Diputados del H. Congreso de la Unión. [en línea]. CAPÍTULO IV, De los Derechos, Facultades y Obligaciones de los Médicos y Personal Sanitario, Artículo 166 Bis 18. https://bit.ly/33HilfH

Makoul, G., Arntson, P., Shofield, T. (1995). Health promotion in primary care: physician-patient communication and decision making about prescription medications. SocSci Med, 41(9). https://bit.ly/3FibIyx

Martínez-Salgado, C. (2012). El muestreo en investigación cualitativa. Principios básicos y algunas controversias. Ciênc. saúde coletiva, Rio de Janeiro, 17 (3).

Mejía, E. A. y Romero, Z., H. (2017). La relación médico paciente: el desarrollo para una nueva cultura médica. Revista Médica Electrónica, 39 (Supl. 1), 832-842. https://bit.ly/3GWCPPY 
Montoya, R., Schmidt, J., García-Caro, M. P., Guardia, P., Cabezas, J. L., Cruz, F. (2010). Influencia del diagnóstico y el pronóstico en el sufrimiento del enfermo terminal. Med Pal, 17, 24-30.

Moreno, S. N. (2019). Educación en dolor crónico: ¿para el paciente o con el paciente? Pasos para establecer una alianza terapéutica. Revista dolor, 8 (2), 76-84. https://bit.ly/32dQwv6

Monte, E. B, Morillo, V. R., Navarro, H., Querd, C. J. y Pere, V. (2019). Importancia de la adherencia al tratamiento de la hepatitis C con antivirales de acción directa. Gastroenterología y Hepatología, 42 (19), 14-19. https://doi.org/10.1016/S0210-5705(20)30183-7

Rodríguez, A. M. (2008). Relación médico-paciente. La Habana: Editorial Ciencias Médicas.

Sánchez, A. D y Contreras, O Y. (2014). La relación médico-paciente y su importancia en la práctica médica. Rev Cub Med Mil, 43 (4),528-533.

Souza, Y. V., Gomes, R., Dos Santos, V., Machado, R., Machado, D. (2020). Percepción de los pacientes sobre su relación con los médicos. Rev. Bioét, 28 (2), 332-343. https://doi.org/10.1590/1983-80422020282395

Torío, D. J., García, T. C. (1997). Relación médico-paciente y entrevista clínica I: opinión y preferencia de los usuarios. Revista Atención Primaria, 19 (1), 18-26. https://bit.ly/3yLVbjT

Van Manen, M. (1990). Researching lived experience: Human science for an action sensitive pedagogy. London, Ontario, Canada: Althouse Press.

Williams, R. J. (2005). Manual de ética médica. Asociación Médica Mundial. https://bit.ly/32oumGc

\section{Cómo citar este trabajo}

Ordóñez Vázquez, N. A., \& Monroy Nasr, Z. (2021). La relación médico paciente vista desde la experiencia de los familiares de enfermos de cáncer en etapa terminal. Apuntes De Bioética, 4(2), 107-123. https://doi.org/10.35383/apuntes.v4i2.657

\section{Financiación}

El presente artículo no cuenta con financiación específica de agencias de financiamiento en los sectores público o privado para su desarrollo y/o publicación.

\section{Agradecimientos}

Al Conacyt y al proyecto DGAPA-PAPIIT IN400319 por el apoyo proporcionado para llevar a cabo esta investigación.

De manera muy especial a los participantes que accedieron a compartir sus experiencias, sin ellas no habría sido posible realizar este trabajo.

\section{Conflicto de interés}

Los autores del artículo declaran no tener ningún conflicto de intereses en su realización.

(C) Los autores. Este artículo es publicado por la Revista Apuntes de Bioética del Instituto de Bioética, Universidad Católica Santo Toribio de Mogrovejo. que permite el uso no comercial, distribución y reproducción en cualquier medio, siempre que la obra original sea debidamente citada. 